\title{
PECAM-1 is involved in BCR/ABL signaling and may downregulate imatinib-induced apoptosis of Philadelphia chromosome-positive leukemia cells
}

\author{
NAN WU, TETSUYA KUROSU, GAKU OSHIKAWA, TOSHIKAGE NAGAO and OSAMU MIURA \\ Department of Hematology, Graduate School of Medical and Dental Sciences, \\ Tokyo Medical and Dental University, Bunkyoku, Tokyo 113-8519, Japan
}

Received September 28, 2012; Accepted November 16, 2012

DOI: 10.3892/ijo.2012.1729

\begin{abstract}
PECAM-1 (CD31) is an immunoreceptor tyrosinebased inhibitory motif (ITIM)-containing surface glycoprotein expressed on various hematopoietic cells as well as on endothelial cells. PECAM-1 has been shown to play roles in regulation of adhesion, migration and apoptosis. The BCR/ABL fusion tyrosine kinase is expressed in chronic myeloid leukemia and Philadelphia-positive $\left(\mathrm{Ph}^{+}\right)$acute lymphoblastic leukemia cells, and its inhibition by the clinically used tyrosine kinase inhibitors imatinib or dasatinib induces apoptosis of these cells. In the present study, we demonstrate that PECAM-1 is tyrosine phosphorylated in its ITIM motifs in various BCR/ABL-expressing cells including primary leukemia cells. Studies using imatinib and dasatinib as well as transient expression experiments in $293 \mathrm{~T}$ cells revealed that PECAM-1 was phosphorylated directly by BCR/ABL, which was enhanced by the imatinib-resistant E255K and T315I mutations, or partly by the Src family tyrosine kinases, including Lyn, which were activated dependently or independently on BCR/ABL. We also demonstrate by using a substrate trapping mutant of SHP2 that tyrosine phosphorylated PECAM-1 binds SHP2 and is a major substrate for this tyrosine phosphatase in BCR/ABL-expressing cells. Overexpression of PECAM-1 in BCR/ABL-expressing cells, including K562 human leukemia cells, enhanced cell adhesion and partially inhibited imatinib-induced apoptosis involving mitochondria depolarization and caspase-3 cleavage, at least partly, in an ITIM-independent manner. These data suggest that PECAM-1 may play a role in regulation of apoptosis as well as adhesion of BCR/ABL-expressing cells to modulate their imatinib sensitivity and would be a possible candidate for therapeutic target in $\mathrm{Ph}^{+}$leukemias.
\end{abstract}

Correspondence to: Professor Osamu Miura, Department of Hematology, Graduate School of Medical and Dental Sciences, Tokyo Medical and Dental University, 1-5-45 Yushima, Bunkyoku, Tokyo 113-8519, Japan

E-mail: miura.hema@tmd.ac.jp

Key words: PECAM-1, BCR/ABL, imatinib, apoptosis, leukemia

\section{Introduction}

PECAM-1, also known as CD31, is a $130-\mathrm{kDa}$ glycoprotein member of the immunoglobulin (Ig)-superfamily of type I transmembrane cell adhesion molecules, which is expressed widely on hematopoietic cells as well as on endothelial cells $(1,2)$. PECAM-1 has a 118 amino-acid cytoplasmic tail that contains 2 immunoreceptor tyrosine-based inhibition motifs (ITIMs) that encompass Y663 and Y686 of human PECAM-1. The ITIMs become tyrosine phosphorylated mainly by the Src family tyrosine kinases in response to various stimuli and recruit several SH2-domain containing signaling molecules, including the protein-tyrosine phosphatase SHP2 and the Src family tyrosine kinases. By coupling with these and various other signaling molecules, PECAM-1 is implicated in modulation of intracellular signaling mechanisms regulating a variety of cellular events, including integrin activation, chemotaxis, apoptosis and cell adhesion (1-4). Recent studies on PECAM-1 deficient mice have further revealed that it plays a regulatory role in SDF-1-induced migration of hematopoietic stem cells and megakaryocytes to the bone marrow vascular niche $(5,6)$. The hematopoietic cytokine IL-3 has been shown to induce tyrosine phosphorylation of PECAM-1 in hematopoietic cells (7). However, its significance in the signal transduction mechanisms by which this hematopoietic cytokine regulates proliferation and apoptosis of cells is still unknown. PECAM-1 is also expressed on various types of leukemias, including acute myeloid leukemia (AML) (8), acute lymphoblastic leukemia (ALL) (9), and chronic lymphocytic leukemia (CLL) $(10,11)$, and has been implicated in prognosis of CLL, although it remains controversial. Thus, PECAM-1 is expected to play important roles in regulation of hematopoiesis and in leukemogenesis, possibly through modulation of apoptosis, cell adhesion and migration, although the molecular mechanisms involved have not been explored.

The BCR/ABL fusion protein is encoded by the fusion gene generated by a reciprocal $\mathrm{t}(9 ; 22)(\mathrm{q} 34 ; \mathrm{q} 11.2)$ chromosomal translocation causing the Philadelphia chromosome $(\mathrm{Ph})$, which is the molecular signature of chronic myeloid leukemia (CML) and is also observed in $30-40 \%$ of ALL $(12,13)$. BCR/ABL is a tyrosine kinase that is constitutively activated and confers survival and proliferation advantages on hematopoietic cells, thus directly contributing to leukemogenesis. CML cells 
express the $\mathrm{p} 210$ form of BCR/ABL, and $\mathrm{Ph}$-positive $\left(\mathrm{Ph}^{+}\right)$ ALL cells mostly express the p190 form, which is generated by the difference in location of gene fusion. Imatinib, a tyrosine kinase inhibitor that blocks the catalytic activity of $\mathrm{BCR} / \mathrm{ABL}$, has demonstrated unprecedented efficacy for treatment of CML or $\mathrm{Ph}^{+}$ALL (12-14). However, the resistance to imatinib may develop in significant portions of patients under treatment, especially in those with CML in advanced stages or with $\mathrm{Ph}^{+}$ALL mostly due to the emergence of mutations in the BCR/ABL kinase domain, including the most prevalent E255K and T315I mutations. We previously showed that the E255K or T315I mutant possessed increased in vitro kinase activities as well increased ability to induce phosphorylation of itself and several cellular substrates when expressed in COS7 cells or in hematopoietic $\mathrm{BaF} 3$ cells as compared with unmutated (native) BCR/ABL (15-17). The increases in transformation abilities for these mutants have also been reported $(18,19)$. The Src family tyrosine kinases are also activated in BCR/ABL-dependent or independent ways and may confer imatinib resistance on these leukemic cells (20-22). To develop more efficient therapeutic strategies against $\mathrm{Ph}^{+}$leukemias, it is very important to gain more insights into the molecular mechanisms involved in imatinib resistance of these leukemias.

In the present study, we show that PECAM-1 is heavily tyrosine phosphorylated on its ITIMs in BCR/ABL-expressing cells, including primary $\mathrm{Ph}^{+}$leukemic cells, at least partly dependent on the BCR/ABL kinase activity. Tyrosine phosphorylated PECAM-1 is physically associated with the SHP2 tyrosine phosphatase and most likely acted as a major substrate for SHP2 in these cells. Intriguingly, tyrosine phosphorylation of PECAM-1 as well as its physical association with SHP2 was enhanced by the imatinib-resistance E255K or T315I mutation. Moreover, overexpression of PECAM-1 enhanced cell adhesion and downregulated imatinib-induced apoptosis on BCR/ ABL-expressing hematopoietic cells. These results suggest that PECAM-1 is involved in BCR/ABL-mediated signaling and may enhance the anti-apoptotic effect.

\section{Materials and methods}

Cells and reagents. A clone of murine IL-3-dependent $\mathrm{BaF} 3$ cells transfected with a BCR/ABL cDNA under the control of a tetracycline-inducible promoter, Ton.B210 and the parental control clone, Ton.BaF, were kindly provided by Dr G. Daley (23). Ton.B210 cells were cultured in 10\% fetal calf serum (FCS) containing RPMI-1640 medium supplemented either with 10\% Wehi3B conditioned medium as the source of IL-3 or with $1 \mu \mathrm{g} / \mathrm{ml}$ doxycycline, which induces the expression of BCR/ABL. Ton.B210/E255K or Ton.B210/T315I cells (16), which inducibly express BCR/ABL with the E255K or T315I mutation, respectively, and 32Dcl 3 cells expressing BCR/ABL, Ton.32Dp210 (17), were described previously. The human CML cell line K562 was obtained from the Riken Cell Bank (Ibaraki, Japan). TMD-5 cells, a double $\mathrm{Ph}^{+}$ALL-derived cell line expressing the $\mathrm{p} 190$ form of $\mathrm{BCR} / \mathrm{ABL}$, were kindly provided by Dr S. Tohda (24). Leukemic blasts were isolated from patients with CML myeloid crisis, $\mathrm{Ph}^{+} \mathrm{ALL}$, or $\mathrm{Ph}^{+}$biphenotypic acute leukemia as described previously (16). Written informed consent was provided according to the Declaration of Helsinki, and the study was approved by the ethics committee of Tokyo Medical and Dental University. PLAT-A (25), an amphotropic virus packaging cell line, and 293T (26), a human embryonic kidney cell line, were kindly provided by Dr T. Kitamura and Dr S. Yamaoka, respectively, and maintained in Dulbecco's modified Eagle's medium (DMEM) supplemented with 10\% FCS.

Imatinib was kindly provided by Novartis (Basel, Switzerland). Dasatinib and sorafenib were purchased from Toronto Research Chemicals Inc. (Toronto, Canada) and LKT Laboratories (St. Paul, MN), respectively. Doxycycline and fibronectin were from Calbiochem (San Diego, CA) and Gibco-BRL (Grand Island, NY), respectively. $\mathrm{DiOC}_{6}$ was purchased from Invitrogen (Carlsbad, CA).

Antibodies against PECAM-1 (SC1506), Lyn (SC15), CrkL (SC319), SHP2 (SC280), BCR (SC885), and phosphoY694-STAT5 (SC9359) were purchased from Santa Cruz Biotechnology (Santa Cruz, CA). An anti-phosphotyrosine monoclonal antibody (4G10, 05-321) as well as antibody against Gab2 (06-967) was purchased from Millipore (Billerica, MA). Antibodies against phospho-Y416-Src (9359) and cleaved caspase-3 (9661) were purchased from Cell Signaling Technology (Beverly, MA). Antibodies against phospho-Y396-Lyn (1645) and $\beta$-actin were purchased from Epitomics Inc. (Burlingame, CA) and Sigma, respectively.

Expression plasmids. Expression plasmids for BCR/ABL, pcDNA3-BCR/ABL, and that for the 56-kDa form of Lyn, pXM-LynA, were described previously $(27,28)$. Retrovirus vectors, pRevTRE and pMIG (Addgene plasmid 9044), were obtained from Clontech (Palo Alto, CA) and Addgene (Cambridge, MA), respectively. pMXs-IG (29) was kindly provided by Dr T. Kitamura. Expression plasmids for wild-type PECAM-1 and its mutant with Y663F and Y686F mutations in the ITIM motives, PECAM-1-ITIM (-), in pcDNA3.0 vector were kindly provided by Dr D. Newman $(30,31)$. The coding regions for wild-type and mutant PECAM-1 were subcloned from these plasmids into retroviral vectors pREV-TRE (HindIII/EcoRV), pMIG $(E c o$ RI) and pXMs-IG (EcoRI) using the restriction enzymes in parentheses. Expression plasmids for wild-type and substrate-trapping mutant of SHP2, pIRES2-EGF-SHP2-Wt and -DA (Addgene plasmids 12283 and 12286) (32), respectively, were obtained from Addgene. The coding sequences for SHP2-Wt and -DA were excised from these plasmids using XhoI and SmaI and subcloned into pREV-TRE to give pREV-SHP2-Wt and -DA. An expression plasmid for BCR/ABL, pTetP210, was kindly provided by Dr G. Daley (23). The coding sequence for BCR/ABL was excised from pTetP210 using EcoRI and subcloned into pRxZiN obtained from the Riken Gene Bank to give pRxP210.

Transfection and infection. For transient expression in 293T cells, cells were transfected with indicated plasmids using the Lipofectamine reagent (Invitrogen) according to the manufacturer's instructions. Cells were harvested $48 \mathrm{~h}$ after transfection for immunoprecipitation and immunoblotting.

To obtain BaF3 cells constitutively expressing BCR/ABL, Ton.BaF cells were infected with the recombinant retrovirus obtained from PLAT-A transfected with pRxP210, as described previously (33). Infected cells were cultured in medium lacking IL-3, and a clone expressing BCR/ABL at a high level was selected by limited dilution to give Ton.Bp210-8. This cell line was subsequently transduced again with pRev-PECAM-1, 
-ITIM (-), or pRevTRE and selected in medium containing hygromycin. Cells were used for subsequent experiments after expression of PECAM-1 or PECAM-1-ITIM (-) was confirmed by immunoblotting. To obtain Ton.B210 cells overexpressing wild-type SHP2 or the D425A mutant, Ton.B210 cells were transduced with pRev-SHP2-Wt or -DA, respectively, and selected in medium containing hygromycin. To obtain Ton.32Dp210 or K562 cells overexpressing PECAM-1 or PECAM-1-ITIM (-), these cells were transduced by the retrovirus vectors in pMXs-IG for pMIG, as described above. GFP-positive cells were sorted by flow cytometry, and expression of PECAM-1 or its mutant as well as BCR/ABL was confirmed by immunoblotting.

Immunoprecipitation and immunoblotting. Cells were lysed and subjected to immunoprecipitation and immunoblotting as described previously (34). The results shown are representative of experiments repeated at least three times.

Analyses of cell viability, apoptosis, caspase-3 cleavage, and mitochondrial membrane potential $\left(\Delta \psi_{m}\right)$. Cell viability was assessed by counting viable and non-viable cell numbers by the trypan blue dye exclusion method. Flow cytometric analysis of cell cycle and apoptosis was performed as described previously (16). Flow cytometric analysis of caspase-3 cleavage was performed using specific antibodies against cleaved caspase-3 as described previously (17). For analysis of $\Delta \psi_{\mathrm{m}}$, cells were stained with $\mathrm{DiOC}_{6}$ (Invitrogen) and analyzed by flow cytometry as described previously (16).

Cell adhesion assay. Adhesion assays were performed essentially as described previously $(35,36)$. In brief, cells were labeled with $5 \mu \mathrm{M}$ BCECF/AM (Dojindo, Kumamoto, Japan) and plated on wells coated with $5 \mu \mathrm{g} / \mathrm{ml}$ fibronectin for $30 \mathrm{~min}$ at $37^{\circ} \mathrm{C}$. Adherent cells were measured by Cytofluor II fluorescent plate reader (PerSeptive Biosystems, Foster City, CA). After subtraction of background cell binding to bovine serum albumin-coated wells, the percentage of adherent cells was determined by dividing the fluorescence intensity of the adherent cells by that of the initial cell input.

\section{Results}

PECAM-1 is tyrosine phosphorylated in primary $\mathrm{Ph}^{+}$leukemic and TMD-5 cells in a manner dependent on the BCR/ABL kinase activity. To examine possible involvement of PECAM-1 in BCR/ABL-mediated signaling, we first examined whether it is tyrosine phosphorylated in primary $\mathrm{Ph}^{+}$leukemic cells. As shown in Fig. 1A, PECAM-1 was conspicuously phosphorylated on tyrosine in $\mathrm{Ph}^{+}$biphenotypic acute leukemia or ALL cells, which was mostly abolished by treatment with the tyrosine kinase inhibitor imatinib or dasatinib. Essentially the same results were obtained with primary leukemic cells from another patient with $\mathrm{Ph}^{+} \mathrm{ALL}$ expressing the p190 form of BCR/ABL and a patient with CML in myeloid blastic crisis expressing the p210 form of BCR/ABL (Fig. 1B and C, respectively). We also examined a $\mathrm{Ph}^{+} \mathrm{ALL}$ cell line expressing the p190 form of BCR/ABL, TMD-5, and found that PECAM-1 was also tyrosine phosphorylated in these cells and was dephosphorylated after treatment with imatinib (Fig. 1D and E). These results suggest that PECAM-1 is a substrate of both p190 and p210 forms of BCR/ABL in these leukemic cells.
PECAM-1 is tyrosine phosphorylated partly through the Src kinases and is a major substrate of SHP 2 in BCR/ABL-expressing cells. We next examined murine model hematopoietic cell lines $32 \mathrm{Dcl} 3$ and $\mathrm{BaF} 3$ engineered to express BCR/ABL. As shown in Fig. 2A and B, PECAM-1 was tyrosine phosphorylated also in these cells, which was completely dephosphorylated by dasatinib. However, imatinib only partially inhibited tyrosine phosphorylation of PECAM-1 in these cells. Because dasatinib, but not imatinib, also inhibits the Src family tyrosine kinases in addition to BCR/ABL, we examined the activation specific tyrosine phosphorylation of these kinases. As shown in Fig. 2C, western blot analysis with an antibody specific for Lyn activation showed that imatinib drastically inhibited activation of Lyn in BCR/ABL-expressing BaF3 cells. However, examination with an antibody that detects activation of the various Src family kinases revealed that some of the activated kinases were resistant to imatinib (Fig. 2C). On the other hand, dasatinib strongly inhibited activation of the Src family kinases including Lyn. These data suggest that Lyn is activated by BCR/ABL in these cells, while some of the other Src family members are constitutively activated independent of BCR/ABL. We also examined the well-established BCR/ABL substrates STAT5 and CrkL in these cells (12). Similar to PECAM-1, tyrosine phosphorylation of CrkL, which was examined by the mobility shift assay, was also abrogated by dasatinib but only partially inhibited by imatinib (Fig. 2C). By contrast, imatinib abrogated tyrosine phosphorylation of STAT5 in BaF3 cells expressing BCR/ABL. These results suggest that the tyrosine phosphorylation of PECAM-1 as well as CrkL, but not that of STAT5, is mediated at least partly by the Src family kinases activated independent of BCR/ABL in these cells.

Wenext examined the possibility that tyrosine phosphorylated PECAM-1 is a substrate for the SHP2 tyrosine phosphatase in these cells, because SHP2 forms a physical complex with tyrosine phosphorylated PECAM-1 in various types of cells $(4,37)$. For this purpose, we overexpressed wild-type SHP2 or its dominant-negative, substrate-trapping mutant SHP-2-D425A $(7,32,38)$ in Ton.B210 cells. As shown in Fig. 2D and E, overexpression of SHP2-D425A, but not wild-type SHP2, profoundly enhanced tyrosine phosphorylation of PECAM-1 and SHP2. Although SHP2 was found to form a complex with PECAM-1 as well as Gab2 in these cells, tyrosine phosphorylation of PECAM-1, but not that of Gab2, that was associated with SHP2 was drastically enhanced by overexpression of SHP2-D425A (Fig. 2E). Tyrosine phosphorylation of SHP2 was also prominently increased in SHP-2-D425A-expressing Ton.B210 cells. These results suggest that PECAM-1 is a major binding partner and substrate of SHP2 in BCR/ABL-expressing cells.

PECAM-1 is tyrosine phosphorylated on ITIM by BCR/ABL and Lyn in 2937 cells. To examine further the mechanisms of tyrosine phosphorylation of PECAM-1 induced by BCR/ABL, we next examined it in transiently transfected $293 \mathrm{~T}$ cells. As shown in Fig. 3A, co-expression of BCR/ABL induced tyrosine phosphorylation of wild-type PECAM-1 but not that of PECAM-1-ITIM (-) with the mutated ITIM motives (Y663F, $\mathrm{Y} 686 \mathrm{~F})$, thus indicating that $\mathrm{BCR} / \mathrm{ABL}$ induces tyrosine phosphorylation of one or both of these ITIM motives. In accordance with a previous report (39), a smaller 120-kDa form of PECAM-1, which most likely represents a differently glycosylated form, was unambiguously observed in transfected cells. 


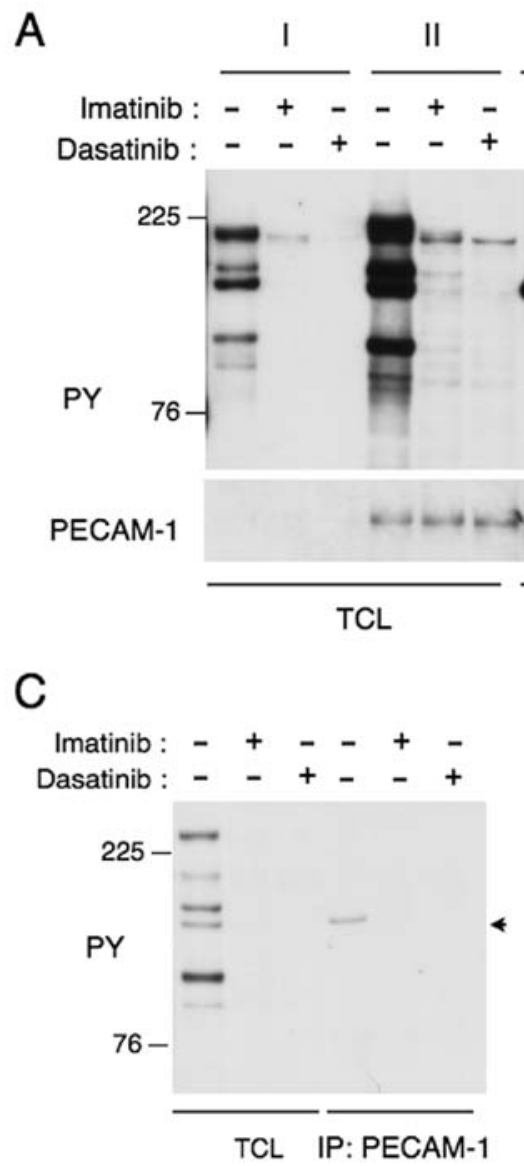

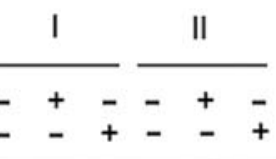
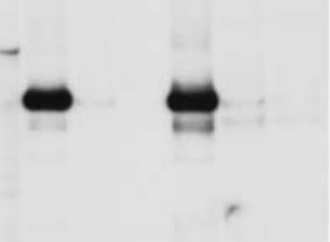

.

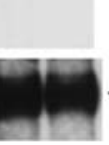

P: PECAM-1
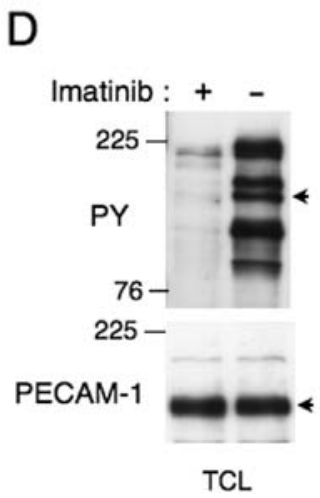

B Imatinib: -++-+

Dasatinib: $\quad$ _ + -+

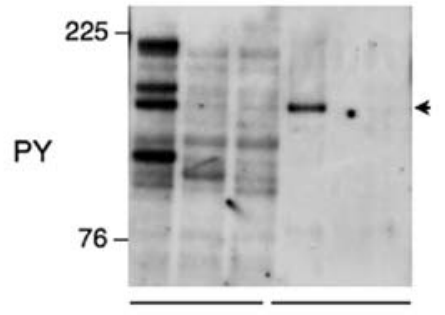

TCL IP: PECAM-1

E

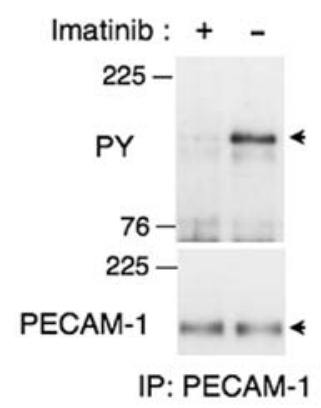

Figure 1. Tyrosine phosphorylation of PECAM-1 in primary $\mathrm{Ph}^{+}$leukemic and TMD-5 cells in a manner dependent on the BCR/ABL kinase activity. (A) Primary leukemic cells from $\mathrm{Ph}^{+}$acute biphenotypic leukemia (I) and ALL (II) patients expressing the p190 form of BCR/ABL were untreated or treated for $2 \mathrm{~h}$ with $10 \mu \mathrm{M}$ imatinib or $0.5 \mu \mathrm{M}$ dasatinib, as indicated. Cells were lysed and subjected to immunoprecipitation with anti-PECAM-1. Total cell lysates (TCL) and immunoprecipitates were then subjected to western blot analysis using anti-phosphotyrosine (PY) and anti-PECAM-1 antibodies, as indicated. The position of PECAM-1 is indicated by an arrowhead. (B) Primary leukemic cells from a $\mathrm{Ph}^{+}$ALL patient were untreated or treated for $2 \mathrm{~h}$ with $5 \mu \mathrm{M}$ imatinib or $5 \mathrm{nM}$ dasatinib, as indicated and analyzed. (C) Primary leukemic cells from a CML patient in blastic phase were untreated or treated for $2 \mathrm{~h}$ with $10 \mu \mathrm{M}$ imatinib or $500 \mathrm{nM}$ dasatinib, as indicated, and analyzed. (D and E) TMD-5 Ph ALL cells were treated with $2 \mu \mathrm{M}$ imatinib for $2 \mathrm{~h}$ or left untreated, as indicated. (D) Total cell lysates (TCL) and (E) anti-PECAM-1 immunoprecipitates were analyzed.

Because Lyn was activated by BCR/ABL and the Src family kinases were implicated in induction of tyrosine phosphorylation of PECAM-1 in BaF3 cells (Fig. 2B and C), we next examined the ability of Lyn to phosphorylate PECAM-1. When co-expressed in $293 \mathrm{~T}$ cells, Lyn induced a robust tyrosine phosphorylation of PECAM-1, which was more conspicuously observed than that induced by BCR/ABL (Fig. 3B and C). Lyn also failed to induce phosphorylation of PECAM-1-ITIM (-). These results are consistent with the idea that the Src kinases including Lyn may partly mediate tyrosine phosphorylation of PECAM-1 on the ITIM motives in cells expressing BCR/ABL.

Tyrosine phosphorylation of PECAM-1 is enhanced by the E255K or T315I imatinib-resistant mutation in BCR/ABL. E255K and T315I are the most predominant mutations of $\mathrm{BCR} / \mathrm{ABL}$ causing imatinib resistance in patients and may increase the kinase activity or change the substrate preferences of BCR/ABL $(15,18)$. Thus, we next examined tyrosine phosphorylation of PECAM-1 in cells expressing BCR/ABL harboring these mutations. As shown in Fig. 4A, PECAM-1 was more prominently tyrosine phosphorylated in $\mathrm{BaF} 3$ cells expressing the E255K or T315I mutant as compared with cells expressing native BCR/ABL. Moreover, as shown in Fig. 4B, SHP2 physically associated with PECAM-1 more prominently in cells expressing the E255K or T315I mutant as compared with cells expressing native $\mathrm{BCR} / \mathrm{ABL}$, while these mutants had less significant effects on complex formation between SHP2 and Gab2. Tyrosine phosphorylation of STAT5 was also enhanced, though not as significantly as that of PECAM-1, in cells expressing the E255K or T315I mutant (Fig. 4C).

We next examined the effect of dasatinib on tyrosine phosphorylation of PECAM-1 in BaF3 cells expressing the T315I mutant, which is totally resistant to dasatinib as well as imatinib but sensitive to the multi-kinase inhibitor sorafenib $(17,22)$. As shown in Fig. 4D, dasatinib or sorafenib partially inhibited tyrosine phosphorylation of PECAM-1. It was confirmed that sorafenib, in contrast to dasatinib, showed inhibitory effect on autophosphorylation of BCR/ABL or phosphorylation of its substrate STAT5 in these cells (Fig. 4E), thus indicating it partially inhibited the T315I mutant in these cells. In 293T cells, the T315I mutant also induced tyrosine phosphorylation of PECAM-1 much more prominently than native BCR/ABL, which was abolished by sorafenib but not affected by dasatinib and correlated with autophosphorylation of BCR/ABL (Fig. 4F 
A

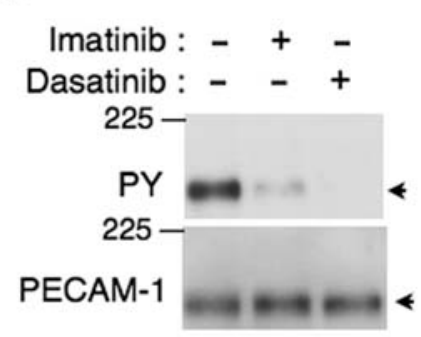

IP: PECAM-1
B

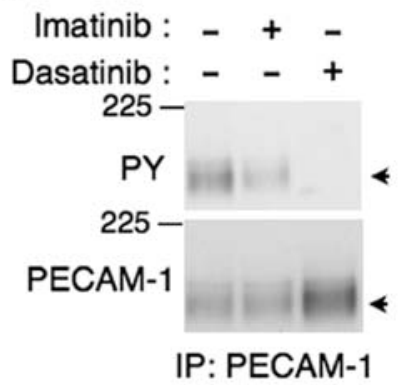

C

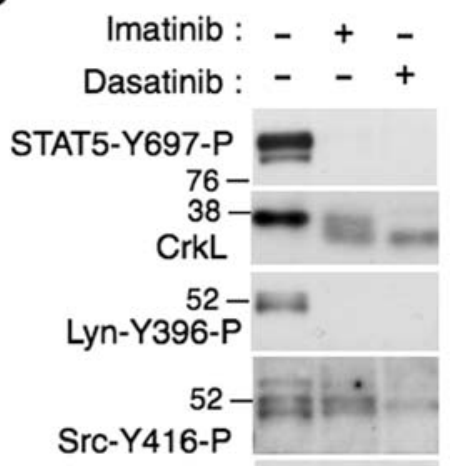

D

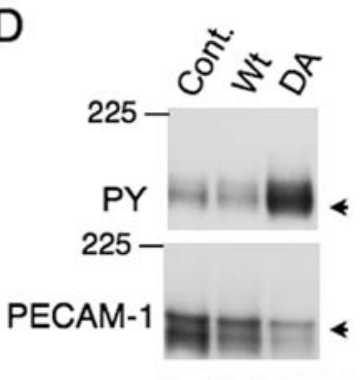

E

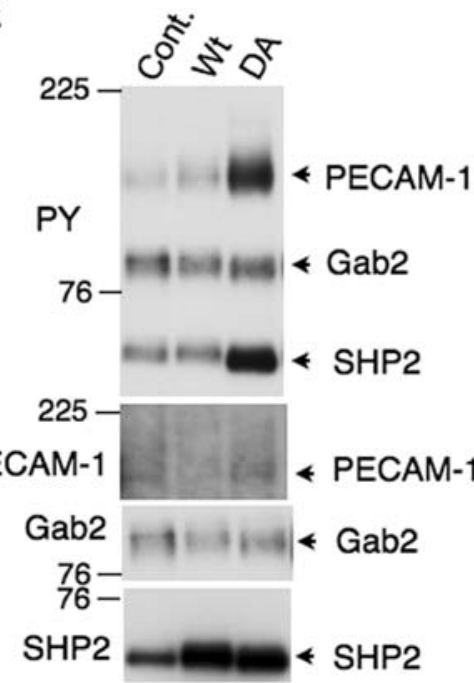

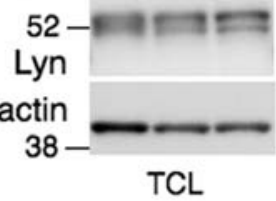

IP: SHP2

Figure 2. Involvement of BCR/ABL, the Src family kinases and SHP2 in regulation of PECAM-1 tyrosine phosphorylation in various BCR/ABL-expressing cell lines. (A) Ton.32Dp210 cells were treated with $10 \mu \mathrm{M}$ imatinib or $0.5 \mu \mathrm{M}$ dasatinib for $6 \mathrm{~h}$ or left untreated as control, as indicated, and analyzed. (B and C) Ton. $\mathrm{B} 210$ cells, a clone of BaF3 cells inducibly expressing BCR/ABL, were cultured in the presence of doxycycline and treated with $10 \mu \mathrm{M}$ imatinib or $0.5 \mu \mathrm{M}$ dasatinib for $2 \mathrm{~h}$ or left untreated as control, as indicated. Immunoprecipitates with anti-PECAM-1 or total cell lysates (TCL) were analyzed. (D and E) Ton.B210 cells overexpressing SHP2 (Wt) or its substrate-trapping D425A mutant (DA) and vector-control cells (Cont) were lysed and immunoprecipitated with (D) anti-PECAM-1 or (E) anti-SHP2, as indicated, for analysis.

A

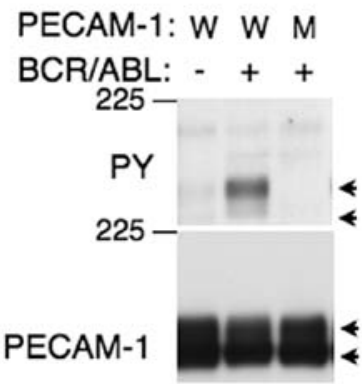

IP: PECAM-1
B

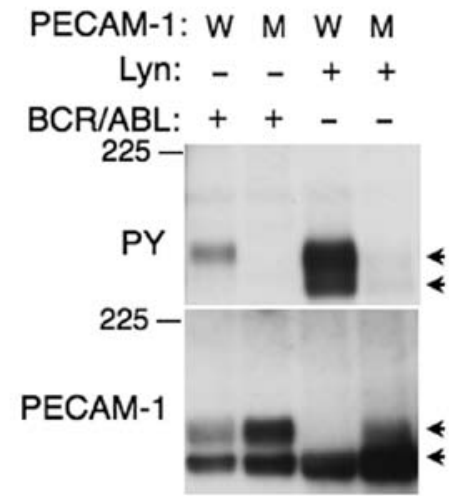

IP: PECAM-1

C

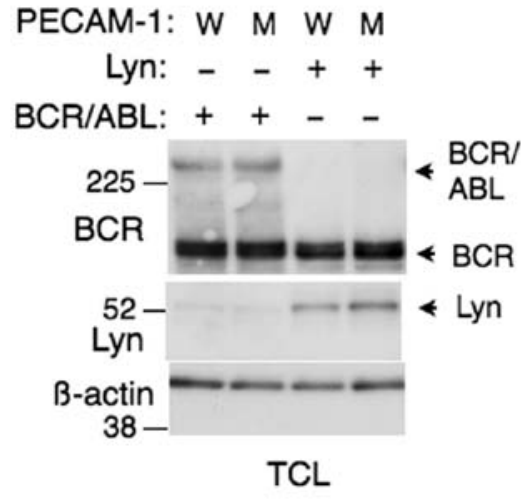

Figure 3. PECAM-1 is tyrosine phosphorylated on ITIMs by BCR/ABL or Lyn in 293T cells. (A) 293T cells were transiently transfected with plasmids coding for BCR/ABL and wild-type PECAM-1 (W) or PECAM-1-ITIM (-) (M), as indicated. Immunoprecipitates with anti-PECAM-1 was analyzed. Positions of PECAM-1 are indicated by arrowheads. (B and C) 293T cells were transfected with plasmids coding for BCR/ABL and Lyn as well as wild-type PECAM-1 (W) or PECAM-1-ITIM (-) (M), as indicated. Immunoprecipitates with anti-PECAM-1 and total cell lysates (TCL) were subjected to western blot analysis using indicated antibodies. Positions of relevant proteins are indicated. 
A

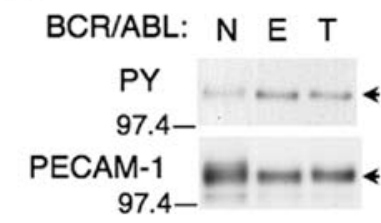

B
D

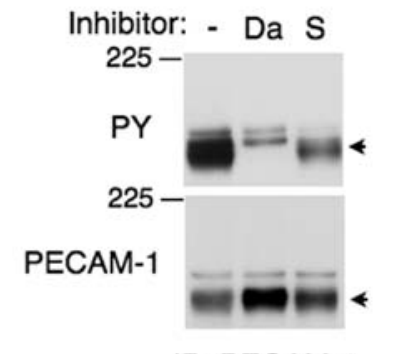

IP: PECAM-1

E

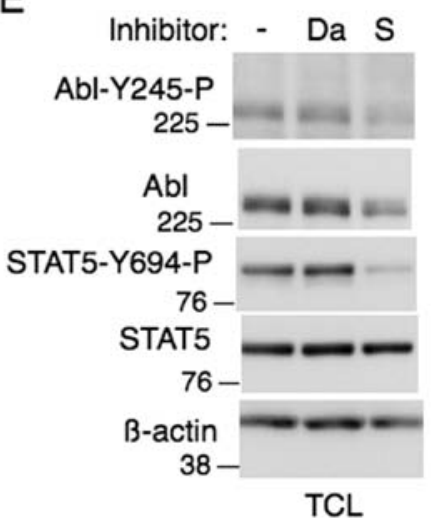

$\mathrm{F}$

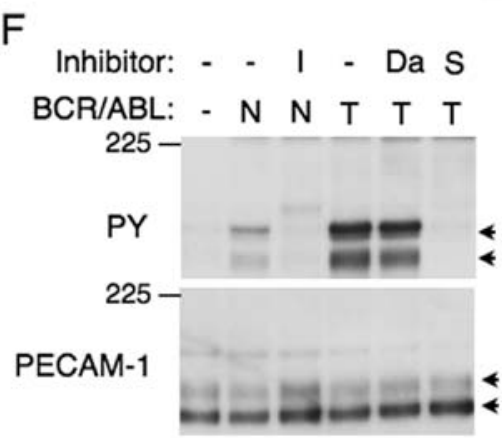

IP: PECAM-1

G

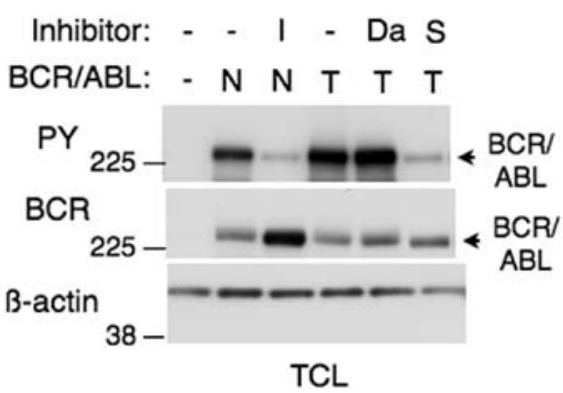

Figure 4. PECAM-1 is more prominently tyrosine phosphorylated in cells expressing the E255K or T315I mutant BCR/ABL. (A, B and C) Ton.B210 (N), Ton.B210/E255K (E), or Ton.B210/T315I (T) cells, cultured in the presence of doxycycline, were lysed. Immunoprecipitates with (A) anti-PECAM-1 or (B) anti-SHP2 as well as (C) total cell lysates (TCL) were subjected to western blot analysis using indicated antibodies. The position of PECAM-1 is indicated by an arrowhead. Positions of other proteins are also indicated. (D and E) Ton.B210/T315I cells were treated with $0.5 \mu \mathrm{M}$ dasatinib (Da) or $10 \mu \mathrm{M}$ sorafenib (S) for $2 \mathrm{~h}$ or left untreated as control, as indicated, and analyzed. ( $\mathrm{F}$ and G) 293T cells were transiently transfected with plasmids coding for native BCR/ABL (N) or the T315I mutant (T), as indicated. Cells were treated with $3 \mu \mathrm{M}$ imatinib (I), $0.5 \mu \mathrm{M}$ dasatinib (Da), or $10 \mu \mathrm{M}$ sorafenib (S) or left untreated, as indicated, for $5 \mathrm{~h}$ before analysis.

and $\mathrm{G})$. The increase in autophosphorylation of the T315I mutation as compared with native BCR/ABL was not so prominent as compared with that in PECAM-1 phosphorylation. These results suggest that the tyrosine phosphorylation of PECAM-1 is mediated both directly by the T315I BCR/ABL mutant and by the Src family kinases in BaF3 cells and exclusively by $\mathrm{BCR} / \mathrm{ABL}$ in $293 \mathrm{~T}$ cells.

Overexpression of PECAM-1 enhances cell adhesion and downregulates imatinib-induced apoptosis in $B C R / A B L$-expressing cells in an ITIM-independent manner. To examine the cellular effects of PECAM-1 in $\mathrm{Ph}^{+}$leukemic cells, we next examined the human CML K562 cell line, which expresses endogenous PECAM-1 at a barely detectable level
(Fig. 5A). As in other BCR/ABL-expressing cells, PECAM-1 overexpressed in K562 cells was tyrosine phosphorylated, which was moderately inhibited or abolished by imatinib or dasatinib, respectively (Fig. 5B). On the other hand, K562 cells overexpressing PECAM-1-ITIM (-) showed a very low level of PECAM-1 phosphorylation, thus suggesting that the ITIM motives are mainly tyrosine phosphorylated also in these $\mathrm{Ph}^{+}$ leukemic cells.

We first examined the possible effect of PECAM-1 on cell adhesion. As shown in Fig. 5C, adhesion of K562 to fibronectin-coated plate was enhanced by overexpression of PECAM-1 or PECAM-1-ITIM (-). These data are in agreement with the previous reports that PECAM-1 may play a role in regulation of integrin activation and cell adhesion in various cell types $(3,37)$. 
A

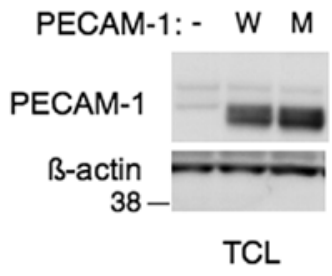

B

Inhibitor: - I Da -

PECAM-1: W W W M

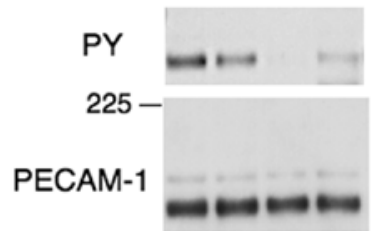

IP: PECAM-1
C

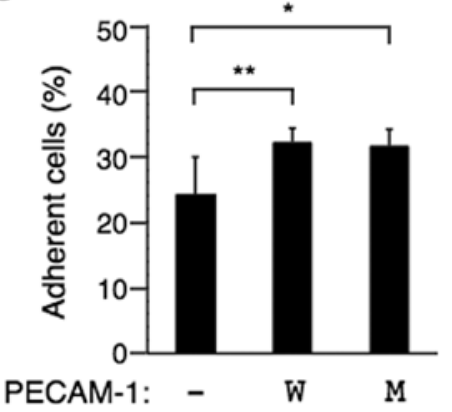

Figure 5. Overexpression of PECAM-1 in human leukemic K562 cells enhances cell adhesion. (A) K562 cells overexpressing wild-type PECAM-1 (W) or PECAM-1-ITIM (-) (M) as well as vector-control cells (-) were lysed. Cellular lysates (TCL) were subjected to western blot analysis with indicated antibodies. (B) K562 cells overexpressing wild-type PECAM-1 (W) or PECAM-1-ITIM (-) (M) were treated for $6 \mathrm{~h}$ with $5 \mu$ M imatinib (I) or $10 \mathrm{nM}$ dasatinib (Da) or left untreated (-), as indicated, and immunoprecipitates with anti-PECAM-1 were analyzed. The position of PECAM-1 is indicated by an arrowhead. (C) K562 cells overexpressing wild-type PECAM-1 (W) or PECAM-1-ITIM (-) (M) as well as vector-control cells (-) were fluorescently labeled and allowed to adhere to wells coated with $5 \mu \mathrm{g} / \mathrm{ml}$ fibronectin for $30 \mathrm{~min}$. The extent of cell adhesion was quantitated as described in Materials and methods. The data represent the mean $\pm \mathrm{SD}$ and representative of experiments repeated three times. The asterisks indicate statistically significant differences determined by Student's $t$-test [ $\left.{ }^{*} \mathrm{p}<0.05,{ }^{* *} \mathrm{p}<0.01(\mathrm{n}=6)\right]$.

A

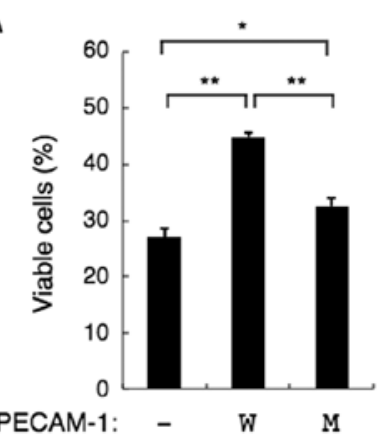

B

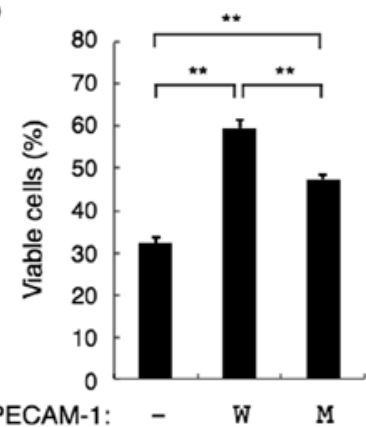

D
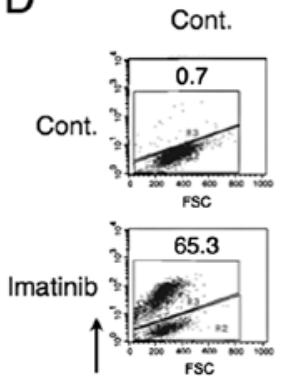

Cleaved Caspase-3
PECAM1 PECAM1-ITIM (-)
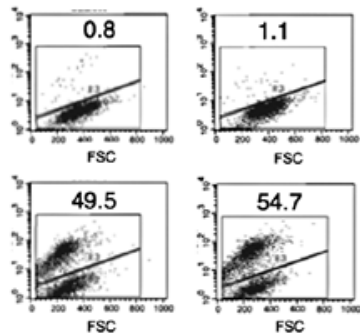

C
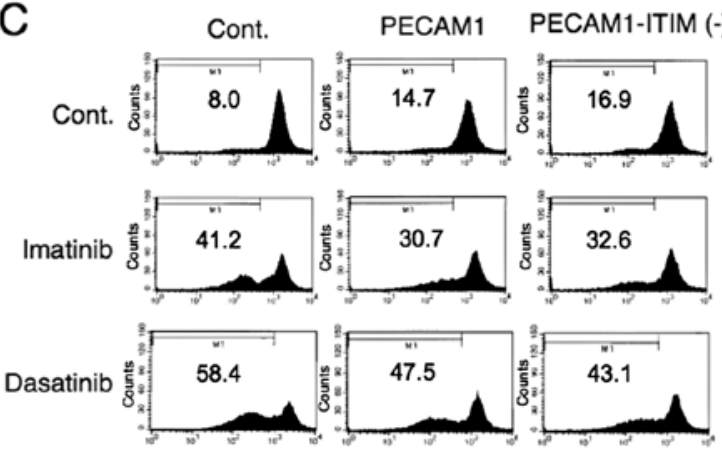

$\longrightarrow$ DiOC6 $\left(\Delta \psi_{\mathrm{m}}\right)$

$\mathrm{E}$
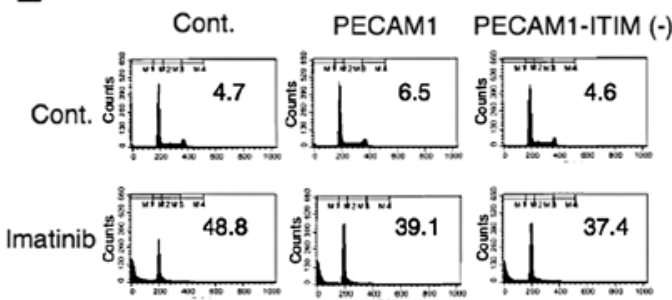

$\longrightarrow$ DNA content

Figure 6. Overexpression of PECAM-1 partially inhibits imatinib-induced apoptosis in BCR/ABL-expressing cells in an ITIM-independent manner (A and B) BCR/ABL-expressing 32D (A) or K562 (B) cells overexpressing wild-type PECAM-1 (W) or PECAM-1-ITIM (-) (M) as well as vector-control cells at $1 \times 10^{5} / \mathrm{ml}$ were cultured with $3 \mu \mathrm{M}$ imatinib for (A) 36 or (B) $48 \mathrm{~h}$, and viability was determined after trypan blue staining. The data represent the mean \pm SD of triplicate samples and representative of experiments repeated three times. ${ }^{*} \mathrm{p}<0.05,{ }^{* *} \mathrm{p}<0.005(\mathrm{n}=3)$. (C) K562 cells overexpressing PECAM-1 or PECAM-1-ITIM $(-)$ and vector-control cells were treated for $36 \mathrm{~h}$ with $0.6 \mu \mathrm{M}$ imatinib or $2 \mathrm{nM}$ dasatinib or left untreated, as indicated, and analyzed for $\Delta \psi_{\mathrm{m}}$ by flow cytometry using DiOC $_{6}$. Percentages of cells showing loss of $\Delta \psi_{\mathrm{m}}$ are indicated. (D) K562 cells indicated were treated for $36 \mathrm{~h}$ with $0.6 \mu \mathrm{M}$ imatinib or left untreated, as indicated, and analyzed for cleavage of caspase-3 by flow cytometry. Percentages of cells showing cleavage of caspase-3 are indicated. (E) K562 cells indicated were treated for $36 \mathrm{~h}$ with $3 \mu \mathrm{M}$ imatinib or left untreated, as indicated, and analyzed for cellular DNA content by flow cytometry. Percentages of apoptotic cells sub-G1 DNA content are indicated.

We next examined the possibility that PECAM-1 may affect the sensitivity of $\mathrm{Ph}^{+}$leukemic cells to the tyrosine kinase inhibitors, because PECAM-1 has been implicated in prevention of apoptosis in various types of cells (40-44). As shown in
Fig. 6A and B, the decline in viability induced by imatinib was downregulated by overexpression of PECAM-1 or, to a lesser degree, by that of PECAM-1-ITIM (-) in BCR/ABL-expressing 32D cells or in K562 cells, respectively. Moreover, PECAM-1 
or its mutant was found to partially prevent depolarization of $\Delta \psi_{\mathrm{m}}$ in K562 cells treated with imatinib or dasatinib (Fig. 6C). In accordance with this, PECAM-1 also partially protected K562 cells from imatinib-induced cleavage of caspase-3 (Fig. 6D). It was finally confirmed that PECAM-1 or its mutant decreased the number of K562 cells with sub-G1 DNA content, a hallmark of cells undergoing apoptosis, after treatment with imatinib (Fig. 6E). Similarly, overexpression of PECAM-1 or its mutant in 32D cells partially downregulated depolarization of $\Delta \psi_{\mathrm{m}}$, cleavage of caspase-3, and appearance of cells with sub-G1 DNA content induced by imatinib (data not shown). Overexpression of PECAM-1 and its mutant showed comparable anti-apoptotic effects in these repeated experiments in K562 and 32D cells. These data suggest that PECAM-1 may partially protect BCR/ABL-expressing leukemic cells treated with the tyrosine kinase inhibitors from activation of mitochondria-mediated apoptotic pathway leading to caspase activation and DNA fragmentation, at least partly, in an ITIM-independent manner.

\section{Discussion}

We have found that PECAM-1 was heavily tyrosine phosphorylated in all the four $\mathrm{Ph}^{+}$leukemic blast samples we examined, including those from biphenotypic AL, ALL and CML-BC patients, the effect was drastically inhibited by imatinib, thus indicating its phosphorylation was mediated by BCR/ABL (Fig. 1). Further studies indicated that tyrosine phosphorylation of PECAM-1 was exclusively mediated by BCR/ABL in 293T cells but was additionally mediated through the Src family kinases, including Lyn, that are activated constitutively or by $\mathrm{BCR} / \mathrm{ABL}$ in various hematopoietic cell lines, such as BaF3, 32D and K562 cells (Figs. 2-5). In this regard, it is noteworthy that BCR/ABL-independent activation of Lyn has been implicated in development of imatinib-resistance in patients with mutation-negative BCR/ABL (21). Although tyrosine phosphorylation of PECAM-1 ITIMs per se may not be required for the anti-apoptotic effect of PECAM-1 in imatinib-treated leukemic cells as discussed below, it is tempting to speculate that interaction of Lyn with PECAM-1 might be involved in acquisition of resistance in these cases, which needs to be addressed in future studies. Previous studies have shown that PECAM-1 is expressed in various types of leukemic cells and implicated its expression in development of the central nervous system involvement of ALL (9), emigration of AML cells from the bone marrow by transendothelial migration (8), and in determination of prognosis of CLL (10). However, the tyrosine phosphorylation status of PECAM-1 has not been examined in these leukemic cells. Thus, its examination in various leukemic cells may shed more light on the significance of PECAM-1 in pathogenesis and prognosis of leukemias.

It is well established that PECAM-1 recruits SHP2 through interaction between its tyrosine phosphorylated ITIMs and the SH2 domains of the tyrosine phosphatase and activates its phosphatase activity $(3,4,37)$. In agreement with this, we observed that tyrosine phosphorylated PECAM-1 formed a complex with SHP2 in BCR/ABL-expressing hematopoietic cells (Fig. 4B). Furthermore, by using the substrate-trapping, loss-of-function mutant of SHP2, we revealed that PECAM-1 is a major substrate of SHP2 in these cells, because PECAM-1 represented an SHP2-associated tyrosine phosphorylated protein that was most significantly enhanced by introduction of SHP2-D425A in BaF3 cells expressing BCR/ABL (Fig. 2E). Previously, Wheadon et al (7) showed that tyrosine phosphorylation of PECAM-1 and Gab2 that bound SHP2 was significantly increased by overexpression of a substrate-trapping C459S mutant of SHP2 in BaF3 cells stimulated with IL-3, thus indicating these proteins are substrates of SHP2 in these cells. Somewhat different from their results, we found that tyrosine phosphorylation of Gab2 that bound SHP2 was not significantly enhanced by overexpression of SHP2-D425A as compared with that of PECAM-1. Therefore, although BCR/ABL and the IL-3 receptor activate similar signaling events involving SHP2 and Gab2, PECAM-1 may play a relatively more significant role as an SHP2-binding substrate as compared with Gab2 in signaling events downstream of BCR/ABL. Previously, SHP2 was postulated to be activated by binding with Gab2 and to play a crucial role in leukemogenesis by BCR/ABL (45-47). The present study raises a possibility that PECAM-1 may also play an important role in activation of the SHP2 signaling events downstream of BCR/ ABL.

Intriguingly, the imatinib-resistant $\mathrm{BCR} / \mathrm{ABL}$ mutants E255K and T315I showed increased ability as compared with native BCR/ABL to induce tyrosine phosphorylation of PECAM-1 when expressed in the murine model hematopoietic cell line BaF3 cells or in 293 T cells (Fig. 4). Furthermore, the E255K and T315I mutations enhanced the complex formation between PECAM-1 and SHP2 (Fig. 4B). The enhanced tyrosine phosphorylation of PECAM-1 by the E255K and T315I mutants may be at least partly due to their enhanced activities (15-17). However, as compared with tyrosine phosphorylation of BCR/ABL and STAT5, that of PECAM-1 was more significantly enhanced by these mutations (Fig. 4A, C, F and G). In this regard, it was previously reported that the imatinib-resistant mutants, including E255K and T315I, exhibited different patterns of substrate phosphorylation as compared with native BCR/ABL, thus suggesting altered substrate specificity and pathway activation (18). Therefore, it is possible that the E255K and T315I mutants may more efficiently interact with and phosphorylate PECAM-1 as compared with native BCR/ABL. Because these mutants are endowed with not only imatinib resistance but also enhanced transforming activities $(18,19)$, a possible significance of PECAM-1 in transforming mechanisms for BCR/ABL including these mutants needs to be addressed in future studies.

The present study revealed that PECAM-1 may enhance the anti-apoptotic effect of BCR/ABL and partially confer imatinib resistance on BCR/ABL-expressing cells by inhibiting the mitochondria-mediated apoptotic mechanisms in a manner at least partly independent of phosphorylation of ITIMs (Fig. 6). Anti-apoptotic effects of PECAM-1 have been previously reported for several types of cells under various conditions, including endothelial cells withdrawn from serum $(41,44)$, hematopoietic cells withdrawn from GM-CSF (42) and an ALL cell line treated with UV irradiation or DNA-damaging chemotherapeutic agents VP16 and AraC $(40,43)$. It was also found that PECAM-1 prevented mitochondria-dependent apoptosis of HEK293T cells induced by overexpression of Bax (43). In these studies, involvement 
of its tyrosine phosphorylation and binding with SHP2 in anti-apoptotic effects has been controversial $(40,43,44)$. Moreover, activation of the PI3K/Akt signaling pathway with upregulation of anti-apoptotic Bcl-2 and Bcl-Xl expression has been implicated in some of these reports $(41,42,44)$, but not in others $(40,43)$. Thus, it is speculated that anti-apoptotic effects of PECAM-1 are mediated through several different mechanisms in different types of cells under various apoptotic stimuli. Intriguingly, PECAM-1 was reported to bind tyrosine-phosphorylated $\beta$-catenin, which was independent of tyrosine phosphorylation of PECAM-1, and to affect its degradation through GSK3 $\beta$-mediated degradation (48). It was also reported that $\beta$-catenin is stabilized through tyrosine phosphorylation by BCR/ABL and may play an essential role in survival of leukemic stem cells expressing BCR/ ABL (49-51). Further studies are in progress in our laboratory to address the possible involvement of $\beta$-catenin in PECAM1-mediated anti-apoptotic mechanisms independent of ITIM phosphorylation. It is also notable that overexpression of PECAM-1 or PECAM-1-ITIM (-) in K562 cells enhanced adhesion of these cells to fibronectin (Fig. 5C), because cell adhesion has been strongly implicated in survival and drug resistance of leukemic cells (52). Taken together with the previous report implicating PECAM-1 in migration of hematopoietic cells to the bone marrow niche (6), where adhesion as well as soluble factors mediate prosurvival effects (52), it is possible that PECAM-1 may play a more prominent role in protection of $\mathrm{Ph}^{+}$leukemic cells from apoptosis in patients treated with imatinib than that expected from the present study. Further studies are warranted to address these possibilities and to elucidate the mechanisms underlying the anti-apoptotic effect of PECAM-1 in $\mathrm{Ph}^{+}$leukemic cells.

\section{Acknowledgements}

We thank Dr Debra Newman for kind advice and generous gifts of experimental materials. We also thank Drs G. Daley, S. Tohda, T. Kitamura, S. Yamaoka and A. Bennett for kind gifts of experimental materials. This study was supported in part by grants from Ministry of Education, Culture, Sports, Science and Technology of Japan (grant nos 21591194, 24591384, 22591030 and 24790966).

\section{References}

1. Newman PJ: The biology of PECAM-1. J Clin Invest 99: 3-8, 1997.

2. Privratsky JR, Newman DK and Newman PJ: PECAM-1: conflicts of interest in inflammation. Life Sci 87: 69-82, 2010.

3. Ilan N and Madri JA: PECAM-1: old friend, new partners. Curr Opin Cell Biol 15: 515-524, 2003.

4. Jackson DE: The unfolding tale of PECAM-1. FEBS Lett 540: 7-14, 2003

5. Dhanjal TS, Pendaries C, Ross EA, et al: A novel role for PECAM-1 in megakaryocytokinesis and recovery of platelet counts in thrombocytopenic mice. Blood 109: 4237-4244, 2007.

6. Ross EA, Freeman S, Zhao Y, et al: A novel role for PECAM-1 (CD31) in regulating haematopoietic progenitor cell compartmentalization between the peripheral blood and bone marrow. PLoS One 3: e2338, 2008.

7. Wheadon H, Edmead C and Welham MJ: Regulation of interleukin-3-induced substrate phosphorylation and cell survival by SHP-2 (Src-homology protein tyrosine phosphatase 2). Biochem J 376: 147-157, 2003.
8. Gallay N, Anani L, Lopez A, et al: The role of platelet/endothelial cell adhesion molecule 1 (CD31) and CD38 antigens in marrow microenvironmental retention of acute myelogenous leukemia cells. Cancer Res 67: 8624-8632, 2007.

9. Akers SM, O'Leary HA, Minnear FL, et al: VE-cadherin and PECAM-1 enhance ALL migration across brain microvascular endothelial cell monolayers. Exp Hematol 38: 733-743, 2010.

10. Ibrahim S, Jilani I, O'Brien S, et al: Clinical relevance of the expression of the CD31 ligand for CD38 in patients with B-cell chronic lymphocytic leukemia. Cancer 97: 1914-1919, 2003.

11. Tonino SH, Spijker R, Luijks DM, van Oers MH and Kater AP: No convincing evidence for a role of CD31-CD38 interactions in the pathogenesis of chronic lymphocytic leukemia. Blood 112: 840-843, 2008

12. Goldman JM and Melo JV: Chronic myeloid leukemia - advances in biology and new approaches to treatment. N Engl J Med 349: 1451-1464, 2003

13. Wong S and Witte ON: The BCR-ABL story: bench to bedside and back. Annu Rev Immunol 22: 247-306, 2004.

14. Gruber F, Mustjoki S and Porkka K: Impact of tyrosine kinase inhibitors on patient outcomes in Philadelphia chromosomepositive acute lymphoblastic leukaemia. Br J Haematol 145: 581-597, 2009.

15. Yamamoto M, Kurosu T, Kakihana K, Mizuchi D and Miura O: The two major imatinib resistance mutations E255K and T315I enhance the activity of BCR/ABL fusion kinase. Biochem Biophys Res Commun 319: 1272-1275, 2004.

16. Kurosu T, Tsuji K, Kida A, Koyama T, Yamamoto M and Miura O: Rottlerin synergistically enhances imatinib-induced apoptosis of BCR/ABL-expressing cells through its mitochondrial uncoupling effect independent of protein kinase C-delta. Oncogene 26: 2975-2987, 2007.

17. Kurosu T, Ohki M, Wu N, Kagechika H and Miura O: Sorafenib induces apoptosis specifically in cells expressing BCR/ABL by inhibiting its kinase activity to activate the intrinsic mitochondrial pathway. Cancer Res 69: 3927-3936, 2009.

18. Griswold IJ, MacPartlin M, Bumm T, et al: Kinase domain mutants of Bcr-Abl exhibit altered transformation potency, kinase activity, and substrate utilization, irrespective of sensitivity to imatinib. Mol Cell Biol 26: 6082-6093, 2006.

19. Skaggs BJ, Gorre ME, Ryvkin A, et al: Phosphorylation of the ATP-binding loop directs oncogenicity of drug-resistant BCR-ABL mutants. Proc Natl Acad Sci USA 103: 19466-19471, 2006.

20. Danhauser-Riedl S, Warmuth M, Druker BJ, Emmerich B and Hallek M: Activation of Src kinases p53/56lyn and p59hck by $\mathrm{p} 210 \mathrm{bcr} / \mathrm{abl}$ in myeloid cells. Cancer Res 56: 3589-3596, 1996.

21. Wu J, Meng F, Kong LY, et al: Association between imatinibresistant BCR-ABL mutation-negative leukemia and persistent activation of LYN kinase. J Natl Cancer Inst 100: 926-939, 2008.

22. Quintas-Cardama A, Kantarjian H and Cortes J: Flying under the radar: the new wave of BCR-ABL inhibitors. Nat Rev Drug Discov 6: 834-848, 2007.

23. Klucher KM, Lopez DV and Daley GQ: Secondary mutation maintains the transformed state in $\mathrm{BaF} 3$ cells with inducible BCR/ABL expression. Blood 91: 3927-3934, 1998.

24. Tohda S, Sakashita C, Fukuda T, Murakami N and Nara N: Establishment of a double Philadelphia chromosome-positive acute lymphoblastic leukemia-derived cell line, TMD5: effects of cytokines and differentiation inducers on growth of the cells. Leuk Res 23: 255-261, 1999.

25. Morita S, Kojima T and Kitamura T: Plat-E: an efficient and stable system for transient packaging of retroviruses. Gene Ther 7: 1063-1066, 2000.

26. DuBridge RB, Tang P, Hsia HC, Leong PM, Miller JH and Calos MP: Analysis of mutation in human cells by using an Epstein-Barr virus shuttle system. Mol Cell Biol 7: 379-387, 1987.

27. Mizuchi D, Kurosu T, Kida A, et al: BCR/ABL activates Rap1 and B-Raf to stimulate the MEK/Erk signaling pathway in hematopoietic cells. Biochem Biophys Res Commun 326: 645-651, 2005.

28. Chin H, Arai A, Wakao H, Kamiyama R, Miyasaka N and Miura O: Lyn physically associates with the erythropoietin receptor and may play a role in activation of the Stat5 pathway. Blood 91: 3734-3745, 1998.

29. Kitamura T, Koshino Y, Shibata F, et al: Retrovirus-mediated gene transfer and expression cloning: powerful tools in functional genomics. Exp Hematol 31: 1007-1014, 2003. 
30. Jackson DE, Kupcho KR and Newman PJ: Characterization of phosphotyrosine binding motifs in the cytoplasmic domain of platelet/endothelial cell adhesion molecule-1 (PECAM-1) that are required for the cellular association and activation of the protein-tyrosine phosphatase, SHP-2. J Biol Chem 272: 24868-24875, 1997.

31. Newman DK, Hamilton C and Newman PJ: Inhibition of antigen-receptor signaling by platelet endothelial cell adhesion molecule-1 (CD31) requires functional ITIMs, SHP-2, and p56(lck). Blood 97: 2351-2357, 2001.

32. Kolli S, Zito CI, Mossink MH, Wiemer EA and Bennett AM The major vault protein is a novel substrate for the tyrosine phosphatase SHP-2 and scaffold protein in epidermal growth factor signaling. J Biol Chem 279: 29374-29385, 2004.

33. Oshikawa G, Nagao T, Wu N, Kurosu T and Miura O: c-Cbl and Cbl-b ligases mediate 17-allylaminodemethoxygeldanamycininduced degradation of autophosphorylated Flt3 kinase with internal tandem duplication through the ubiquitin proteasome pathway. J Biol Chem 286: 30263-30273, 2011.

34. Miura O, Cleveland JL and Ihle JN: Inactivation of erythropoietin receptor function by point mutations in a region having homology with other cytokine receptors. Mol Cell Biol 13: 1788-1795, 1993.

35. Arai A, Nosaka Y, Kanda E, Yamamoto K, Miyasaka N and Miura O: Rapl is activated by erythropoietin or interleukin-3 and is involved in regulation of betal integrin-mediated hematopoietic cell adhesion. J Biol Chem 276: 10453-10462, 2001.

36. Jin A, Kurosu T, Tsuji K, et al: BCR/ABL and IL-3 activate Rap1 to stimulate the B-Raf/MEK/Erk and Akt signaling pathways and to regulate proliferation, apoptosis, and adhesion. Oncogene 25: 4332-4340, 2006.

37. Newman PJ and Newman DK: Signal transduction pathways mediated by PECAM-1: new roles for an old molecule in platelet and vascular cell biology. Arterioscler Thromb Vasc Biol 23: 953-964, 2003.

38. Flint AJ, Tiganis T, Barford D and Tonks NK: Development of 'substrate-trapping' mutants to identify physiological substrates of protein tyrosine phosphatases. Proc Natl Acad Sci USA 94: 1680-1685, 1997.

39. Albelda SM, Muller WA, Buck CA and Newman PJ: Molecular and cellular properties of PECAM-1 (endoCAM/CD31): a novel vascular cell-cell adhesion molecule. J Cell Biol 114: 1059-1068, 1991.

40. Bergom C, Goel R, Paddock C, et al: The cell-adhesion and signaling molecule PECAM-1 is a molecular mediator of resistance to genotoxic chemotherapy. Cancer Biol Ther 5: $1699-1707,2006$.
41. Bird IN, Taylor V, Newton JP, et al: Homophilic PECAM-1(CD31) interactions prevent endothelial cell apoptosis but do not support cell spreading or migration. J Cell Sci 112: 1989-1997, 1999.

42. Ferrero E, Belloni D, Contini P, et al: Transendothelial migration leads to protection from starvation-induced apoptosis in $\mathrm{CD} 34^{+} \mathrm{CD} 14^{+}$circulating precursors: evidence for PECAM-1 involvement through Akt/PKB activation. Blood 101: 186-193, 2003.

43. Gao C, Sun W, Christofidou-Solomidou M, et al: PECAM-1 functions as a specific and potent inhibitor of mitochondrialdependent apoptosis. Blood 102: 169-179, 2003.

44. Limaye V, Li X, Hahn C, et al: Sphingosine kinase-1 enhances endothelial cell survival through a PECAM-1-dependent activation of PI-3K/Akt and regulation of Bcl-2 family members. Blood 105: 3169-3177, 2005.

45. Chan G, Kalaitzidis D and Neel BG: The tyrosine phosphatase Shp2 (PTPN11) in cancer. Cancer Metastasis Rev 27: 179-192, 2008.

46. Scherr M, Chaturvedi A, Battmer K, et al: Enhanced sensitivity to inhibition of SHP2, STAT5, and Gab2 expression in chronic myeloid leukemia (CML). Blood 107: 3279-3287, 2006.

47. Chen J, Yu WM, Daino H, Broxmeyer HE, Druker BJ and Qu CK: SHP-2 phosphatase is required for hematopoietic cell transformation by Bcr-Abl. Blood 109: 778-785, 2007.

48. Biswas P, Canosa S, Schoenfeld D, et al: PECAM-1 affects GSK-3beta-mediated beta-catenin phosphorylation and degradation. Am J Pathol 169: 314-324, 2006.

49. Coluccia AM, Vacca A, Dunach M, et al: Bcr-Abl stabilizes beta-catenin in chronic myeloid leukemia through its tyrosine phosphorylation. EMBO J 26: 1456-1466, 2007.

50. Hu Y, Chen Y, Douglas L and Li S: beta-Catenin is essential for survival of leukemic stem cells insensitive to kinase inhibition in mice with BCR-ABL-induced chronic myeloid leukemia. Leukemia 23: 109-116, 2009.

51. Shang YC, Zhang C, Wang SH, et al: Activated beta-catenin induces myogenesis and inhibits adipogenesis in BM-derived mesenchymal stromal cells. Cytotherapy 9: 667-681, 2007.

52. Meads MB, Hazlehurst LA and Dalton WS: The bone marrow microenvironment as a tumor sanctuary and contributor to drug resistance. Clin Cancer Res 14: 2519-2526, 2008. 